\title{
Assessment of carotid atherosclerotic plaque using 3D motion- sensitized driven-equilibrium prepared rapid gradient echo: a comparative study
}

\author{
Xin Cao ${ }^{1, \#}$, Ye Tang ${ }^{1 \#}$, Lei Pan ${ }^{1 \#}$, Jinming Yang ${ }^{1}$, Yifan Wu ${ }^{1,2}$, Daoying Geng ${ }^{1,2}$, Jun Zhang ${ }^{1,2}$ \\ ${ }^{1}$ Department of Radiology, Huashan Hospital, Fudan University, Shanghai, China; ${ }^{2}$ Institute of Functional and Molecular Medical Imaging, Fudan \\ University, Shanghai, China
}

\#These authors contributed equally to this work and are co-first authors.

Correspondence to: Daoying Geng; Jun Zhang. Department of Radiology, Huashan Hospital, Fudan University, 12 Wulumuqi Middle Road, Shanghai 200040, China. Email: gdy_2019@163.com; zhj81828@163.com.

Background: 3D motion-sensitized driven-equilibrium prepared rapid gradient echo (MERGE) can characterize carotid atherosclerotic plaque morphology and composition. The present study aimed to evaluate its performance by comparing it with reference images and assessing the inter-reader agreement.

Methods: Eighty-four patients were prospectively recruited and scanned with 3D MERGE. Two trained magnetic resonance imaging (MRI) readers measured and calculated the maximum wall thickness (WT), maximum vessel diameter, total vessel area, lumen area, wall area, normalized wall index, plaque volume, intraplaque hemorrhage (IPH) volume, and calcification volume independently. IPH, calcification, mixed calcification, and ulceration were identified. The intraclass correlation coefficient (ICC) with $95 \%$ confidence interval (CI) was used to assess the inter-reader agreement. MERGE performance was assessed in terms of sensitivity, specificity, positive predictive value, negative predictive value, positive likelihood ratio, negative likelihood ratio, kappa value $(\kappa)$, and the results of the Bland-Altman analysis and compared with reference images.

Results: MERGE showed excellent inter-reader agreement (All ICCs >0.90). MERGE and simultaneous non-contrast angiography and intraplaque hemorrhage (SNAP) showed excellent agreement in detecting IPH ( $\kappa=0.938)$ and measuring IPH volume (ICC =0.995; 95\% CI: 0.991-0.997). MERGE and computed tomography angiography (CTA) showed strong consistency in detecting calcification $(\kappa=0.814)$ and mixed calcification ( $\kappa=0.972$ ), and in measuring calcification volume (ICC =0.996; 95\% CI: 0.993-0.997). MERGE and digital subtraction angiography (DSA) showed relatively strong consistency in identifying ulceration $(\kappa=0.737)$.

Conclusions: MERGE showed excellent performance in identifying and measuring IPH and calcification in carotid atherosclerotic plaques. Therefore, MERGE can be a promising imaging approach in atherosclerotic-vulnerable plaque.

Keywords: Motion-sensitized driven-equilibrium prepared rapid gradient echo (MERGE); atherosclerosis; carotid plaque; magnetic resonance imaging (MRI)

Submitted Jul 15, 2020. Accepted for publication Oct 09, 2020.

doi: 10.21037/qims-20-869

View this article at: http://dx.doi.org/10.21037/qims-20-869 


\section{Introduction}

Carotid atherosclerosis is increasingly considered the leading cause of ischemic stroke $(1,2)$. Some specific morphological features of symptomatic carotid plaque, such as plaque burden, plaque size, surface status, and components, are risk factors for stroke (3-6). Clinical studies focusing on the detection and volume assessment of intraplaque hemorrhage (IPH) have demonstrated a strong correlation between IPH and future cerebrovascular events $(7,8)$. Calcification in plaque is categorized as surface, deep, or mixed type, and mixed calcification exists both on the surface and in the plaque's deep area (9). The quantity and configuration of calcification may affect plaque stability $(10,11)$. Irregular plaque surface, particularly ulceration, is a major vulnerability sign, which indicates previous plaque rupture $(12,13)$.

Vulnerable atherosclerotic plaque has been studied extensively using a variety of imaging methods, including ultrasound, digital subtraction angiography (DSA), computed tomography angiography (CTA), and magnetic resonance imaging (MRI). High-resolution vessel wall MRI (VW-MRI) is a promising imaging tool recommended by the American Society of Neuroradiology to quantitatively measure plaque morphology and components, such as plaque burden, surface irregularity, and IPH (14). Simultaneous non-contrast angiography and intraplaque hemorrhage (SNAP) is a highly $\mathrm{T}_{1}$-weighted MRI sequence with a wide coverage of $3 \mathrm{D}$ isotropic inversion recovery and phase-sensitive reconstruction (15). IPH is readily detectable as a high signal on the SNAP image. The interreader agreement for the manual detection of IPH on SNAP is remarkably strong $(16,17)$, and the measurement of IPH volume on SNAP imaging is consistent with that of histology (16).

Several 3D black-blood MRI sequences with high isotropic resolution, high signal-to-noise ratio, and wide coverage have been developed $(15,18)$. Black-blood MRI using motion-sensitized driven-equilibrium prepared rapid gradient echo (MERGE) can provide imaging with improved longitudinal coverage of bilateral carotid arteries simultaneously in a short acquisition time $(18,19)$. Similar to the previous histologically confirmed multi-contrast VWMRI and time-of-flight magnetic resonance angiography, MERGE can also provide comparable performance in carotid atherosclerotic plaque with good inter-reader reproducibility (17). MERGE has isotropic resolution and multiple planar reconstruction capabilities, and may yield a more accurate delineation of plaque surface and imaging of plaque components (12). However, its accuracy and reliability in assessing extracranial carotid atherosclerotic plaque have not been well studied systematically. Therefore, in the present study, we aimed to compare MERGE's diagnostic performance with reference images (SNAP, CTA, and DSA) by assessing IPH, calcification, and ulceration, respectively.

\section{Methods}

\section{Study design and participants}

This study was approved by the Institutional Review Board of Huashan Hospital, Fudan University (Shanghai, China; No. 2013.332). Written informed consent was obtained from either the patient or their legal representative before study entry.

Between April 2019 and July 2020, patients with recent cerebrovascular symptoms (amaurosis fugax, transient ischemic attack, or suspected cerebrovascular ischemia in the anterior circulation within 2 weeks) and atherosclerotic plaque in at least 1 side of the carotid artery identified by ultrasound imaging (intima-media thickness $\geq 1.5 \mathrm{~mm}$ ) were prospectively recruited. The exclusion criteria were as follows: (I) hemorrhagic stroke; (II) cardiogenic stroke; (III) cerebral tumor; (IV) history of carotid endarterectomy (CEA); (V) contraindication to MRI, CTA, or DSA examination; and (VI) dementia or coma. All patients underwent 3D VW-MRI and CTA examination within 3 days before DSA. Patients who could not tolerate or complete all examinations were excluded. Clinical characteristics, including age, sex, body mass index (BMI), history of diabetes mellitus, hypertension, hyperlipidemia, alcoholism, smoking, total cholesterol, high-density lipoprotein cholesterol, low-density lipoprotein cholesterol, triglyceride, lipoprotein (a), and glucose, were collected from medical records.

\section{MRI protocol}

Patients were scanned within 2 weeks after the occurrence of cerebrovascular symptoms. A 3.0-T MR system (Ingenia; Philips Healthcare, The Netherlands) was used with an 8-channel phased-array carotid artery coil (TS Imaging, Beijing, China) and a 20-channel phased-array head and neck joint coil (Philips Healthcare, the Netherlands). After localization, MERGE and SNAP images were obtained using the following parameters: sagittal orientation, centered on the common carotid artery (CCA) bifurcation, 
the scan coverage comprising the $\mathrm{C} 1-\mathrm{C} 7$ segments of the bilateral internal carotid artery (ICA), and the M1-M2 segments of the bilateral middle cerebral artery:

(I) $3 \mathrm{D}$ MERGE: echo time (TE)/repetition time $(\mathrm{TR})=5.2 / 11.0 \mathrm{~ms}$, field of view $(\mathrm{FOV})$ $=230 \times 161 \times 60 \mathrm{~mm}^{3}$, voxel $=0.8 \times 0.8 \times 0.8 \mathrm{~mm}^{3}$, matrix size $=288 \times 200 \times 100$, number of slices $=100$, reconstruction resolution $=0.4 \times 0.4 \times 0.4 \mathrm{~mm}^{3}$, and scan time $=2 ' 51$;

(II) $3 \mathrm{D} \mathrm{SNAP:} \mathrm{TE/TR}=5.0 / 11.0 \mathrm{~ms}, \mathrm{FOV}$ $=230 \times 161 \times 60 \mathrm{~mm}^{3}$, voxel $=0.8 \times 0.8 \times 0.8 \mathrm{~mm}^{3}$, matrix size $=288 \times 200 \times 100$, number of slices $=100$, reconstruction resolution $=0.4 \times 0.4 \times 0.4 \mathrm{~mm}^{3}$, and scan time $=4^{\prime} 28$.

\section{CTA imaging protocol}

CTA imaging was performed in the head-first supine position using a 256-slice-CT scanner (iCT; Philips Healthcare, The Netherlands). A total of $60 \mathrm{~mL}$ (rate: $5 \mathrm{~mL} / \mathrm{s}$ ) contrast medium was injected into the right elbow vein. Tube voltage/current $=120 \mathrm{kV} / 360 \mathrm{~mA}$, section thickness/spacing $=0.90 / 0.45 \mathrm{~mm}$, and voxel $=0.6 \times 0.6 \times 0.3 \mathrm{~mm}^{3}$.

\section{DSA imaging protocol}

All sets of DSA images were obtained using an Infinx/ VC digital angiography system (Toshiba, Tokyo, Japan) operated by experienced neuro-interventionalists. The Seldinger technique was used to obtain images of the ICA, including the anterior-posterior and lateral projections. A total of $7 \mathrm{~mL}$ (rate: $4 \mathrm{~mL} / \mathrm{s}$ ) contrast medium was injected into the carotid artery, and the image acquisition rate was 3.5 frames/s. The suspicious artery was imaged by $240^{\circ}$ rotation, with an image acquisition of 15 frames/s. Matrix size $=1,024 \times 1,024$ and voxel $=0.3 \times 0.3 \times 1.0 \mathrm{~mm}^{3}$.

\section{Image analysis}

A neuro-interventionalist with more than 15 years' work experience reviewed the DSA images and recorded ulcerations by observing the plaque on the best projection at a workstation (AW4.4; GE Healthcare, BOSTON, MA, USA). Some cases with existing surgical indications received a second verification of CEA in the intervention therapy department. For some ambiguous cases, senior doctors' ultrasound diagnosis reports were used as a reference for comprehensive judgment. The final recorded data on plaque ulceration were the reference standard for the present study. ITK-SNAP software v.3.6.0 (University of Pennsylvania, www.itksnap.org/) was used for reformatting the original isotropic coronal MR images to axial and sagittal ones. A neuroradiologist with more than 20 years' work experience reviewed the SNAP and CTA images and performed morphological quantification as the reference standard. SNAP imaging was used to determine the IPH and to measure the IPH volume by software. Plaque calcification and its classification and volume measurement were performed using the CTA image. All MERGE images of carotid arteries were analyzed and measured by 2 trained readers with more than 5 years' experience in plaque imaging. They were both blinded to each other's findings and the reference images. Image quality (IQ) was evaluated for all MR images using a 4-point scale with the following classifications: IQ $=1$, poor quality ( $V W$ and lumen margin and plaque compositions not identifiable); IQ =2, adequate quality (the boundary of $\mathrm{VW}$ can be identified, but the compositional substructure cannot be determined); IQ =3, good quality (minimal motion or flow artifacts, VW, lumen boundary and plaque compositions can be defined); and $\mathrm{IQ}=4$, excellent quality (no artifacts, wall architecture and plaque composition can be depicted clearly in detail) (20). Images with IQ $\leq 2$ were excluded.

An atherosclerotic plaque is defined as a lesion with an intima-media thickness $>1.5 \mathrm{~mm}$ of the $\mathrm{VW}$ on reformatted 3D MERGE axial images. The threshold of $1.5 \mathrm{~mm}$ was chosen according to the correlation between the wall thickness (WT) and the increased stroke risk $(21,22)$. If multiple stenoses were present in a single carotid artery, the section with the most severe stenosis degree was studied for statistical analysis. The maximum WT (Max WT), maximum vessel diameter (Max VD), total vessel area (TVA), and lumen area (LA) were measured at the same crosssection of vessel. Furthermore, the wall area (WA) and normalized wall index (NWI) were automatically calculated to evaluate the plaque burden by using the following formulas:

$$
\begin{aligned}
& W A=T V A-L A \\
& \mathrm{NWI}=\frac{T V A-L A}{\mathrm{TVA}}
\end{aligned}
$$

If plaques were present on both the bilateral extracranial carotid arteries, the carotid artery with the larger NWI value was regarded as the research object. The identification of each plaque composition was based on criteria as follows: IPH is identified as an obvious hyperintense signal on MERGE images (Figure 1), and calcification 

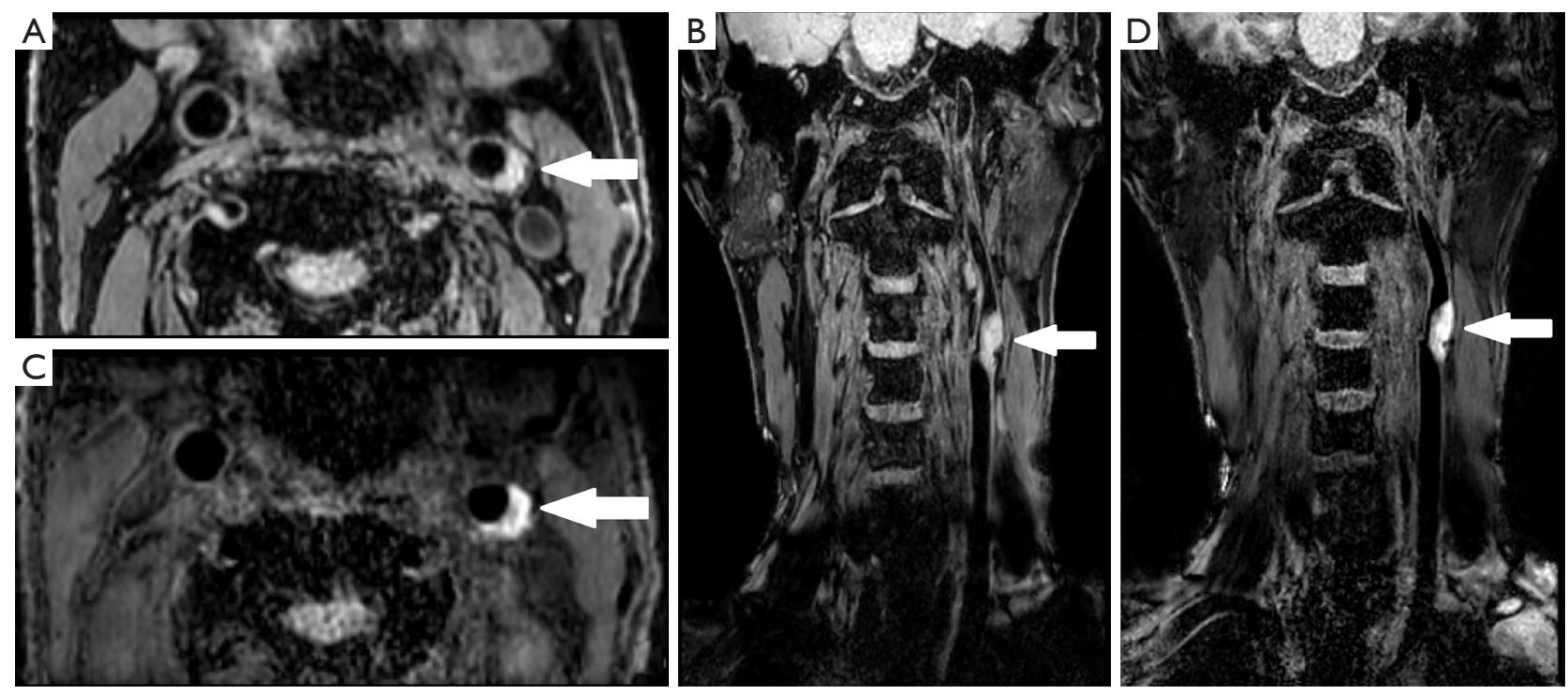

Figure 1 An example of a 71-year-old male patient who had intermittent dizziness, nausea, and weakness of both lower limbs. Bleeding plaque was detected in his left common carotid artery and the bilateral internal carotid artery. High signal on axial (A) and coronal (B) image of 3D motion-sensitized driven-equilibrium prepared rapid gradient echo showed intraplaque hemorrhage (IPH) (white arrows). Obvious high signal on axial (C) and coronal (D) image of 3D simultaneous non-contrast angiography and intraplaque hemorrhage showed IPH (white arrows).

is characterized by areas with a hypointense signal on MERGE images (Figure 2) $(23,24)$. Mixed calcification refers to the calcification distributed on both the surface and deep areas of the plaque (9). Some studies have defined ulceration as the extended lumen into the plaque with a width $>1 \mathrm{~mm}$ or depth $>2 \mathrm{~mm}$ (25-27). Ulceration was observed on multi-dimensional reconstructed images (Figure 3). IPH or calcification volume was measured with ITK-SNAP software. The above morphological identification, measurement, and calculation were performed by 2 image readers independently.

\section{Statistical analysis}

Continuous variables are presented as mean \pm standard deviation (SD), categorical variables as frequencies, and the presence or absence of plaque components was denoted as binary variables. For continuous variables, an agreement among Max WT, Max VD, TVA, LA, plaque volume, IPH volume, and calcification volume between $2 \mathrm{MR}$ image readers was assessed using 2-way random intraclass correlation coefficient (ICC); the consistency test of WA and NWI calculated using formulas was summarized using 2-way random ICC. For categorical variables, the presence or absence of IPH, calcification, mixed calcification, and ulceration, an agreement was summarized using 1-way random ICC. Bias between 2 readers was assessed quantitatively by mean difference \pm standard error. MannWhitney $U$-test was used to test for significant differences between their results. The detection performance of IPH, calcification, mixed calcification, and ulceration were summarized by sensitivity, specificity, positive predictive value, negative predictive value, positive likelihood ratio, negative likelihood ratio, and kappa value $(\kappa)$ by using SNAP, CTA, and DSA results as references. Bland-Altman analysis was used to test the measurement agreements between MERGE and reference images (SNAP and DSA) of the volume of IPH and calcification. All statistical analyses were performed using SPSS software version 23.0 (SPSS IBM, Armonk, New York, USA). $\mathrm{P}<0.05$ was considered statistically significant.

\section{Results}

\section{Patients' clinical characteristics}

Of the 84 patients, 11 were excluded from the analysis due to the following reasons: inadequate image quality (IQ $\leq 2$ ) with severe blood flow artifacts $(n=1)$ or motion artifacts $(n=4)$, one side of the ICA was completely occluded $(n=3)$, and carotid arteries had dissection or dissecting aneurysm 

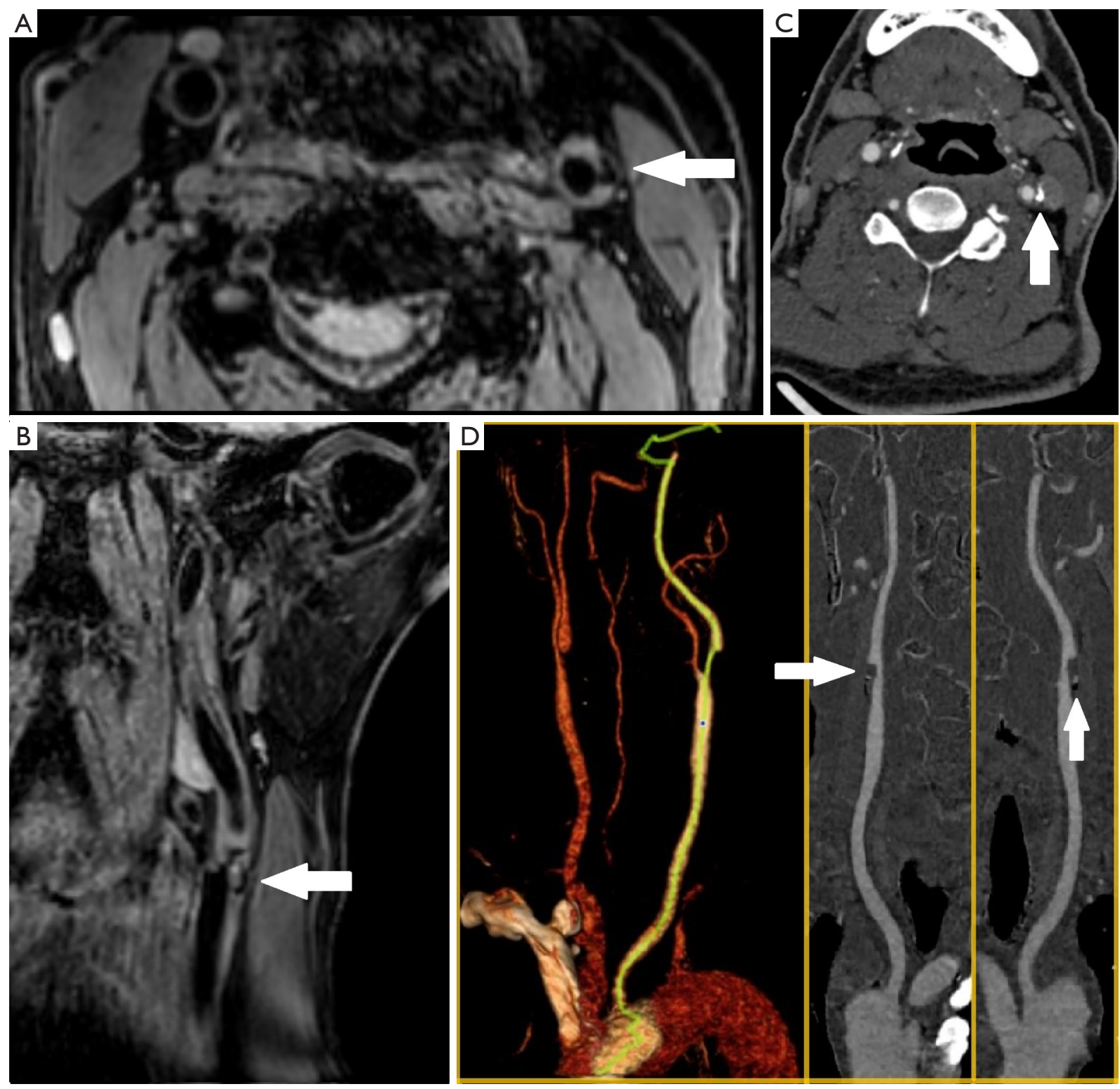

Figure 2 An example of a 63 -year-old male patient who had sudden numbness and fatigue in his right limb. Calcified atherosclerotic plaque was detected in his left common carotid artery. Low signal on axial (A) and coronal (B) image of 3D motion-sensitized driven-equilibrium prepared rapid gradient echo showed calcification (white arrows). Computed tomography angiography clearly showed the shape and distribution of calcification within plaque (C,D) (white arrows); the calcification type of his plaque was mixed.

$(\mathrm{n}=3)$. Of the 73 sets of MERGE images of diseased carotid arteries, 68 (93.15\%) were graded as excellent (IQ =4). The demographic and clinical characteristics of the 73 patients are summarized in Table 1. The mean age of the patients was $65.63 \pm 8.68$ years, and $63(86.30 \%)$ were male. Their mean BMI was $24.11 \pm 2.61 \mathrm{~kg} / \mathrm{m}^{2}$. Of all patients, $47(64.38 \%)$ had hypertension, and 27 (36.98\%) had a smoking history.

\section{Inter-reader agreement on 3D MERGE}

All ICCs for measurements and calculations based on 3D MERGE was $\geq 0.90$, indicating excellent inter-reader reproducibility (Table 2). The ICC for the measurement of plaque volume reached 1.000 . The ICC with the lowest value of 0.907 (0.856-0.941) was the detection of calcification. Differences between some metrics between 2 

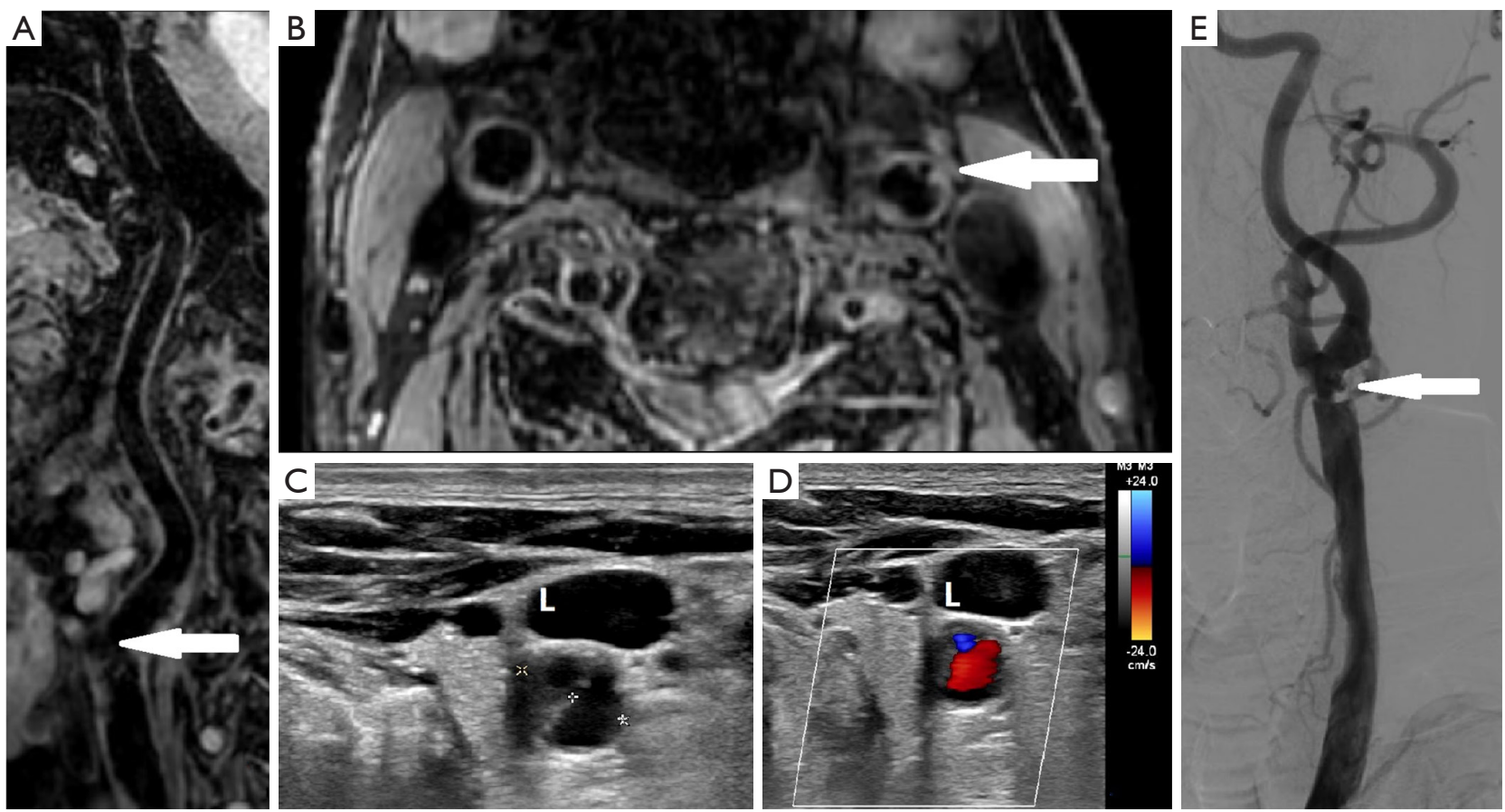

Figure 3 An example of an 81-year-old male patient who had severe stenosis of the left carotid artery with multiple ischemic infarcts in his brain. Ruptured plaque was detected in his left common carotid artery. Plaque surface defect on the curved multi-planar reconstruction image along the lumen (A) and axial image (B) of 3D motion-sensitized driven-equilibrium prepared rapid gradient echo was ulceration (white arrows). Ultrasound image showed a small plaque defect (C), and the blood flow within the ulceration was relatively disordered (D). Small niche displayed by digital subtraction angiography was plaque ulceration (E) (white arrows).

Table 1 Clinical characteristics of the study cohort

\begin{tabular}{lc}
\hline Characteristics & Participants $(\mathrm{n}=73)$ \\
\hline Age, years & $65.00(60.00,72.50)$ \\
Males, $\mathrm{n}(\%)$ & $63(86.30)$ \\
$\mathrm{BMI}, \mathrm{kg} / \mathrm{m}^{2}$ & $23.88(22.17,26.09)$ \\
Medical history, $\mathrm{n}(\%)$ & $20(27.39)$ \\
Diabetes mellitus & $47(64.38)$ \\
Hypertension & $6(8.22)$ \\
Hyperlipidemia & $14(19.17)$ \\
Alcoholism & $27(36.98)$ \\
Smoking & \\
Laboratory examination, mmol/L & $3.72(2.98,4.15)$ \\
Total cholesterol & $1.06(0.92,1.15)$ \\
HDL cholesterol & $2.10(1.73,2.64)$ \\
LDL cholesterol & $1.45(1.04,1.88)$ \\
Triglyceride & $38.50(13.00,161.00)$ \\
Lipoprotein (a) & $5.80(5.20,7.40)$ \\
Glucose &
\end{tabular}

Data are presented as $\mathrm{n}(\%)$ or median (p25, p75). BMI, body mass index; HDL, high density lipoprotein; LDL, low density lipoprotein. readers, such as the measurements of Max WT, Max VD, TVA, LA, and plaque volume, were significant $(\mathrm{P}<0.05)$.

\section{Agreement of IPH between 3D MERGE and 3D SNAP}

In the SNAP images, 23 of $73(31.51 \%)$ plaques were found to have IPH, and the 2 readers each identified 25 and 24 plaques with IPH. They each had 2 and 1 false-positive results for a specificity of $96 \%$ and $98 \%$, respectively. Their sensitivity values were $100 \%$. Kappa test between MERGE and SNAP showed strong consistency $(\kappa=0.938, \mathrm{P}<0.001)$ (Table 3). Bland-Altman plots of IPH volume measurement between MERGE and SNAP suggested that the mean difference was $0.48 \mathrm{~mm}^{3}$, and no significant bias was observed (Figure 4). IPH volume measurements obtained using MERGE and SNAP had a good agreement (ICC $=0.995,95 \%$ CI: 0.991-0.997).

\section{Agreement of calcification between 3D MERGE and CTA}

Of the 73 plaques, 47 (64.38\%) had calcification on CTA 
Table 2 Inter-reader agreement for 73 plaques on 3D MERGE images

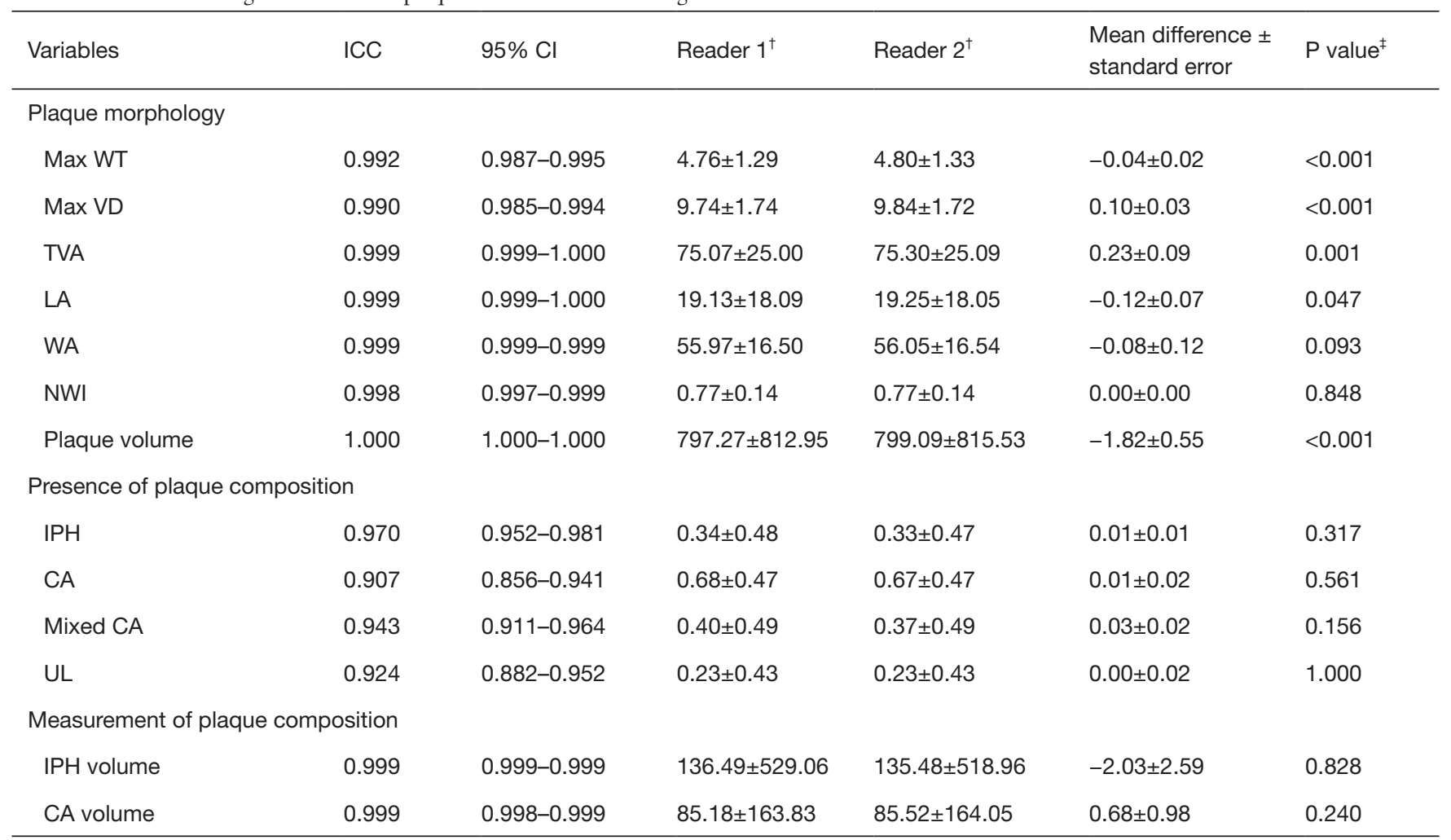

${ }^{\dagger}$, data are presented as mean \pm standard deviation. ${ }^{\ddagger}$, for comparison of mean difference to 0 . CA, calcification; $\mathrm{Cl}$, confidence interval; ICC, intraclass correlation coefficient; IPH, intraplaque hemorrhage; LA, lumen area; Max WT, maximum wall thickness; Max VD, maximum vessel diameter; MERGE, motion-sensitized driven-equilibrium prepared rapid gradient echo; NWI, normalized wall index; SE, standard error; TVA, total vessel area; UL, ulceration; WA, wall area.

Table 3 Diagnostic consistency and performance of 3D MERGE on plaque features

\begin{tabular}{lllllllll}
\hline Features & $\kappa$ & P value & Sensitivity & Specificity & PPV & NPV & PLR & NLR \\
\hline IPH & 0.938 & $<0.01$ & $1.000(0.822-1.000)$ & $0.960(0.851-0.993)$ & $0.920(0.725-0.986)$ & $1.000(0.908-1.000)$ & 25.00 & 0.00 \\
CA & 0.814 & $<0.01$ & $0.979(0.873-0.999)$ & $0.808(0.600-0.927)$ & $0.902(0.778-0.963)$ & $0.955(0.751-0.998)$ & 5.10 & 0.03 \\
Mixed CA & 0.972 & $<0.01$ & $0.967(0.809-0.998)$ & $1.000(0.898-1.000)$ & $1.000(0.854-1.000)$ & $0.977(0.865-0.999)$ & - & 0.03 \\
UL & 0.737 & $<0.01$ & $0.778(0.519-0.926)$ & $0.945(0.839-0.986)$ & $0.824(0.558-0.953)$ & $0.929(0.819-0.977)$ & 14.15 & 0.23 \\
\hline
\end{tabular}

Only the comparison between MR reader 1 and reference were listed because of the strong inter-reader agreement with MR reader 2 . Numbers in parentheses are 95\% confidence intervals. CA, calcification; IPH, intraplaque hemorrhage; $\kappa$, kappa value; MERGE, motionsensitized driven-equilibrium prepared rapid gradient echo; MR, magnetic resonance; NLR, negative likelihood ratio; NPV, negative predictive value; PLR, positive likelihood ratio; PPV, positive predictive value; UL, ulceration.

images, while 30 of 47 had mixed calcification. The 2 readers each identified 51 and 50 plaques with calcification, and 29 and 27 mixed calcifications, respectively. Kappa test showed that the detected calcification and mixed calcification between MERGE and CTA had very strong consistency $(\kappa=0.814,0.972, \mathrm{P}<0.001)($ Table 3$)$. The Bland-
Altman plots of calcification volume measurement between MERGE and CTA suggested that the mean difference was $0.71 \mathrm{~mm}^{3}$, and no significant bias was observed (Figure 4). The calcification volume measurements obtained using MERGE and CTA had a good agreement (ICC $=0.996$, 95\% CI: 0.993-0.997). 

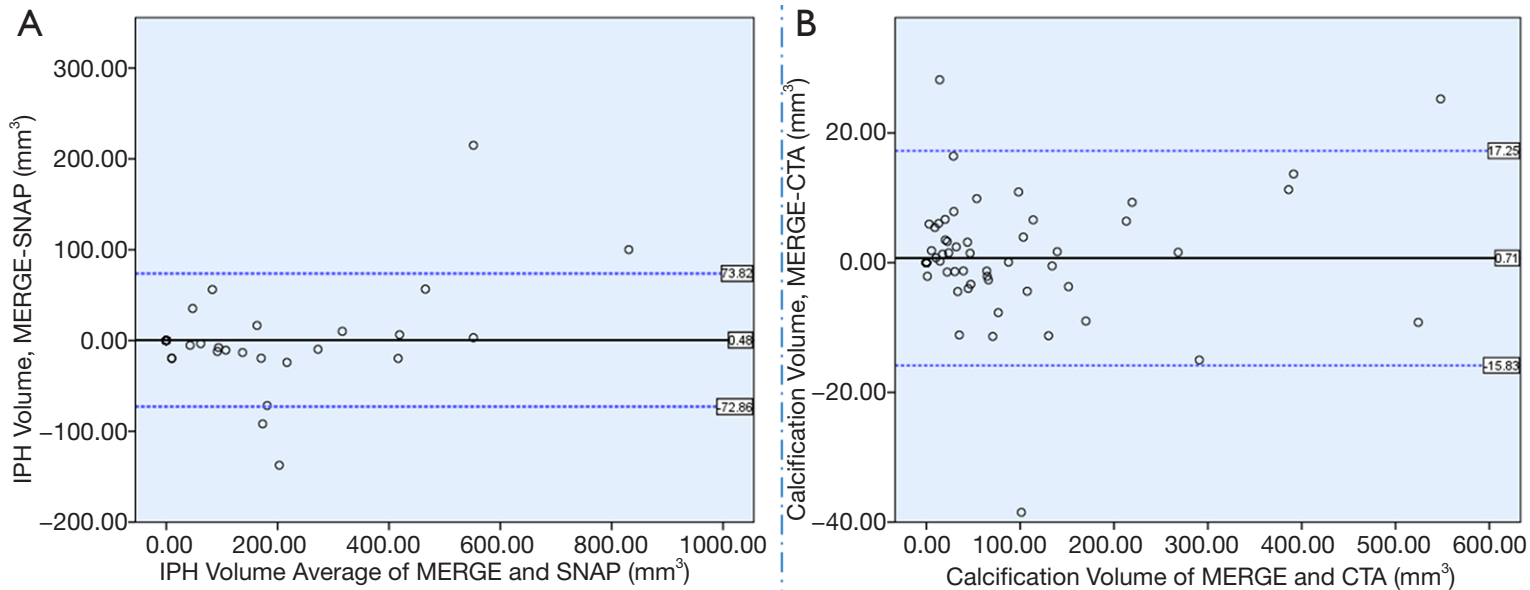

Figure 4 Bland-Altman plots of intraplaque hemorrhage volume measurements between motion-sensitized driven-equilibrium prepared rapid gradient echo (MERGE) image reader 1 and simultaneous non-contrast angiography and intraplaque hemorrhage in symptomatic atherosclerotic plaques (A). Bland-Altman plots of calcification volume measurements between MERGE reader 1 and computed tomography angiography in symptomatic atherosclerotic plaques (B). Solid lines represent the mean difference (overall bias), and dashed lines indicate the $95 \%$ limits of agreement.

\section{Agreement of ulceration between 3D MERGE and DSA}

In the DSA images, 18 of 73 plaques (24.66\%) were found to have ulcerations. Of these, 6 cases were confirmed by the CEA, and 3 cases were confirmed with a combination of CEA and ultrasound diagnostic reports. Both MR image readers identified 17 plaques containing ulcerations. The sensitivity values were $77.78 \%$ and $83.33 \%$, and the specificity values were $94.55 \%$ and $96.36 \%$, respectively. Kappa test showed a relatively strong agreement for the detection of ulceration between MERGE and DSA ( $\kappa=0.737$, $\mathrm{P}<0.001)$ (Table 3).

\section{Discussion}

We compared MERGE performance and references in assessing symptomatic carotid plaques and confirmed the accuracy of MERGE in both morphological measurement and plaque component identification. The present study showed the usefulness of MERGE as a reliable and accurate modality for atherosclerotic plaque imaging.

MERGE showed good inter-observer reproducibility in identifying plaque components and measuring morphological parameters $(17,28,29)$. Slow and stagnant blood flow is eliminated by dephasing the flow spins within each voxel (30). Wei et al. revealed a good inter-reader agreement to identify calcification based on multi-contrast imaging with $\kappa=0.66$ (17), but this result was not as good as ours. It may be because calcification is manifested as an obvious low signal, but the human eye is more sensitive to high signals, which leads to differences in personal subjective vision. Zhao et al. obtained $\kappa=0.95(0.84-0.99)$ for ulceration identification (29), which was better than our result. The observation of ulceration was affected by varying reconstruction habits and doctors' experiences. The systematic differences in Max WT, Max VD, TVA, LA, and plaque volume might be due to the non-circular lumen shape, irregular shape of plaque, and different measurement habits in our study. Some systematic differences in diameter measurements, particularly for large-size vessel segments in other studies were noted (31). Nevertheless, these slight differences had no significant effect on the accuracy of diagnosis.

To the best of our knowledge, the present study is the first attempt to validate MERGE-based IPH detection and volume measurement in symptomatic carotid plaque using SNAP imaging as a reference. IPH shows a high signal on $T_{1}$-weighted sequences due to the short $T_{1}$ relaxation of methemoglobin with super para-magnetism $(15,32)$. Compared with the magnetization prepared rapid gradient echo (33), SNAP is more sensitive to IPH and can provide higher IPH-plaque contrast (Figure 1). Previous studies have reported the strong agreement between the high signal detected on conventional MRI and histologically confirmed IPH (34), but with a systematic underestimation 
of IPH area using histology as the gold standard (24); therefore, SNAP might exhibit higher sensitivity in detecting small IPH lesions in plaque. Qiao et al. found moderate agreement between $T_{1}$ mapping cardiovascular MR and histology in detecting IPH and excellent agreement in measuring the area of IPH (ICC $=0.816,95 \%$ CI: $0.679-0.894)$ with a systematic overestimation (35). Our research demonstrated that MERGE and SNAP show similar performance in detecting IPH. The high signal provides an excellent contrast to yield an accurate delineation of the IPH within the plaque. With its low false-negative rate, MERGE could be useful for screening carotid vulnerable plaque. IPH is a dynamic process (36). VW-MRI, as a non-invasive imaging method that outweighs histological examination in many cases. MERGE can achieve similar detection sensitivity and volume measurement accuracy of IPH as SNAP does, and it has the potential to monitor the natural history of IPH with the half scanning time of the latter sequence.

CTA can display and accurately measure the size, shape, and distribution of calcification (Figure 2). In contrast, MERGE does not require contrast agents and exhibits wide coverage, and the improved spatial resolution of 0.4 $\mathrm{mm}$ results in improved visualization for the detection of small calcifications that would otherwise remain undetected due to partial volume averaging (18). Calcification can be delineated clearly, and mixed calcification can be detected as low signal distributed both on the surface and in the plaque's deep area (Figure 2). The location distribution of calcification may affect the regularity of the plaque surface, which may further influence plaque vulnerability (12). Through the accurate assessment of calcification in plaque by MERGE, the underlying mechanism behind the relationship between mixed calcification and irregular plaque surface is yet to be revealed.

The diagnostic accuracy of non-invasive imaging is usually assessed using DSA as a reference standard. However, the disadvantages of DSA, including the possibility of neurological complications in $0.5-1 \%$ of patients (37), have prompted researchers to consider noninvasive methods as an alternative to DSA. The suboptimal performance of DSA in detecting ulceration of symptomatic plaque has been previously reported (38-40). VW-MRI has high sensitivity, high specificity, and good agreement with DSA for detecting ulceration $(29,31)$. In our study, the plaque ulcerations of some patients were validated by DSA and by histological examination after CEA. We did not perform histological examination for patients who had no surgical indications to comply with the ethical principles, but we determined the presence or absence of plaque ulcerations comprehensively through ultrasound and DSA diagnoses (Figure 3).

Given that MERGE images can be reconstructed using a curved multi-planar reformation algorithm along the lumen or any orientation without tissue distortion, it can directly display the precise details of plaque ulceration (Figure 3) (29). However, the doctor's manual reconstruction may potentially introduce subjective bias. The falsenegative cases of missed small ulcerations were attributed to the limited spatial resolution; calcification adjacent to the lumen may obscure the lumen surface, resulting in the misidentification of false-positive cases (23). It has been reported that bright-blood sequences are highly accurate in detecting ulceration (41); therefore, the combination of bright-blood and black-blood technique may serve as an alternative imaging approach to DSA in identifying plaque ulceration.

The present study has several limitations. First, the morphology and composition of plaque were observed by SNAP, CTA, and DSA as reference images, which were less accurate than a histological gold standard. Although these previously proposed techniques are histologically validated, further histological data are essential to evaluate MERGE's reliability and stability for future work. Second, the target plaque was located either at the CCA bifurcation or at the initial ICA segment, which may cause a certain degree of analysis bias.

\section{Conclusions}

3D MERGE showed good performance in identifying and measuring IPH and calcification in carotid plaques and performed well in the detection of plaque ulceration. It may be a promising alternative imaging approach for histological examination in some clinical studies of carotid atherosclerotic plaques.

\section{Acknowledgments}

Funding: The present study was funded by the National Natural Science Foundation of China (No. 81971598), the Shanghai Shuguang Program (No. 19SG06), Clinical Medicine Research Pilot Project of Shanghai Medical College of Fudan University (No. DGF501022/015), and Shanghai Municipal Commission of Science and Technology (No. 19411951200). 


\section{Footnote}

Conflicts of Interest: All authors have completed the ICMJE uniform disclosure form (available at http://dx.doi. org/10.21037/qims-20-869). The authors have no conflicts of interest to declare.

Ethical Statement: The present study was approved by the Institutional Review Board of Huashan Hospital, Fudan University (No. 2013.332). Written informed consent was obtained from either the patient or their legal representative before study entry.

Open Access Statement: This is an Open Access article distributed in accordance with the Creative Commons Attribution-NonCommercial-NoDerivs 4.0 International License (CC BY-NC-ND 4.0), which permits the noncommercial replication and distribution of the article with the strict proviso that no changes or edits are made and the original work is properly cited (including links to both the formal publication through the relevant DOI and the license). See: https://creativecommons.org/licenses/by-nc-nd/4.0/.

\section{References}

1. GBD 2016 Neurology Collaborators. Global, regional, and national burden of neurological disorders, 1990-2016: a systematic analysis for the Global Burden of Disease Study 2016. Lancet Neurol 2019;18:459-80.

2. Fereydooni A, Gorecka J, Xu J, Schindler J, Dardik A. Carotid Endarterectomy and Carotid Artery Stenting for Patients With Crescendo Transient Ischemic Attacks: A Systematic Review. JAMA Surg 2019;154:1055-63.

3. Moody AR, Murphy RE, Morgan PS, Martel AL, Delay GS, Allder S, MacSweeney ST, Tennant WG, Gladman J, Lowe J, Hunt BJ. Characterization of complicated carotid plaque with magnetic resonance direct thrombus imaging in patients with cerebral ischemia. Circulation 2003;107:3047-52.

4. Saam T, Hatsukami TS, Takaya N, Chu B, Underhill H, Kerwin WS, Cai J, Ferguson MS, Yuan C. The vulnerable, or high-risk, atherosclerotic plaque: noninvasive $\mathrm{MR}$ imaging for characterization and assessment. Radiology 2007;244:64-77.

5. Takaya N, Yuan C, Chu B, Saam T, Underhill H, Cai J, Tran N, Polissar NL, Isaac C, Ferguson MS, Garden GA, Cramer SC, Maravilla KR, Hashimoto B, Hatsukami TS. Association between carotid plaque characteristics and subsequent ischemic cerebrovascular events: a prospective assessment with MRI--initial results. Stroke 2006;37:818-23.

6. Yuan C, Mitsumori LM, Beach KW, Maravilla KR. Carotid atherosclerotic plaque: noninvasive $\mathrm{MR}$ characterization and identification of vulnerable lesions. Radiology 2001;221:285-99.

7. Yamada K, Song Y, Hippe DS, Sun J, Dong L, Xu D, Ferguson MS, Chu B, Hatsukami TS, Chen M, Zhou C, Yuan C. Quantitative evaluation of high intensity signal on MIP images of carotid atherosclerotic plaques from routine TOF-MRA reveals elevated volumes of intraplaque hemorrhage and lipid rich necrotic core. J Cardiovasc Magn Reson 2012;14:81.

8. Teng Z, Sadat U, Brown AJ, Gillard JH. Plaque hemorrhage in carotid artery disease: pathogenesis, clinical and biomechanical considerations. J Biomech 2014;47:847-58.

9. Lin R, Chen S, Liu G, Xue Y, Zhao X. Association Between Carotid Atherosclerotic Plaque Calcification and Intraplaque Hemorrhage: A Magnetic Resonance Imaging Study. Arterioscler Thromb Vasc Biol 2017;37:1228-33.

10. Yang J, Pan X, Zhang B, Yan Y, Huang Y, Woolf AK, Gillard JH, Teng Z, Hui P. Superficial and multiple calcifications and ulceration associate with intraplaque hemorrhage in the carotid atherosclerotic plaque. Eur Radiol 2018;28:4968-77.

11. Kan Y, He W, Ning B, Li H, Wei S, Yu T. The correlation between calcification in carotid plaque and stroke: calcification may be a risk factor for stroke. Int J Clin Exp Pathol 2019;12:750-8.

12. Li J, Li D, Yang D, Hang H, Wu Y, Yao R, Chen X, Xu Y, Dai W, Zhou D, Zhao X. Irregularity of Carotid Plaque Surface Predicts Subsequent Vascular Event: A MRI Study. J Magn Reson Imaging 2020;52:185-94.

13. Yuan J, Usman A, Das T, Patterson AJ, Gillard JH, Graves MJ. Imaging Carotid Atherosclerosis Plaque Ulceration: Comparison of Advanced Imaging Modalities and Recent Developments. AJNR Am J Neuroradiol 2017;38:664-71.

14. Mandell DM, Mossa-Basha M, Qiao Y, Hess CP, Hui F, Matouk C, Johnson MH, Daemen MJ, Vossough A, Edjlali M, Saloner D, Ansari SA, Wasserman BA, Mikulis DJ. Intracranial Vessel Wall MRI: Principles and Expert Consensus Recommendations of the American Society of Neuroradiology. AJNR Am J Neuroradiol 2017;38:218-29.

15. Wang J, Börnert P, Zhao H, Hippe DS, Zhao X, Balu N, Ferguson MS, Hatsukami TS, Xu J, Yuan C, Kerwin WS. Simultaneous noncontrast angiography and intraplaque 
hemorrhage (SNAP) imaging for carotid atherosclerotic disease evaluation. Magn Reson Med 2013;69:337-45.

16. Liu J, Sun J, Balu N, Ferguson MS, Wang J, Kerwin WS, Hippe DS, Wang A, Hatsukami TS, Yuan C. Semiautomatic carotid intraplaque hemorrhage volume measurement using 3D carotid MRI. J Magn Reson Imaging 2019;50:1055-62.

17. Wei H, Zhang M, Li Y, Zhao X, Canton G, Sun J, Xu D, Zhou Z, Chen S, Ferguson MS, Hatsukami TS, Li R, Yuan C. Evaluation of 3D multi-contrast carotid vessel wall MRI: a comparative study. Quant Imaging Med Surg 2020;10:269-82.

18. Balu N, Yarnykh VL, Chu B, Wang J, Hatsukami T, Yuan C. Carotid plaque assessment using fast 3D isotropic resolution black-blood MRI. Magn Reson Med 2011;65:627-37.

19. Murata K, Murata N, Chu B, Watase H, Hippe DS, Balu N, Sun J, Zhao X, Hatsukami TS, Yuan C. Characterization of Carotid Atherosclerotic Plaques Using 3-Dimensional MERGE Magnetic Resonance Imaging and Correlation With Stroke Risk Factors. Stroke 2020;51:475-80.

20. Underhill HR, Yarnykh VL, Hatsukami TS, Wang J, Balu N, Hayes CE, Oikawa M, Yu W, Xu D, Chu B, Wyman BT, Polissar NL, Yuan C. Carotid plaque morphology and composition: initial comparison between 1.5- and 3.0-T magnetic field strengths. Radiology 2008;248:550-60.

21. Kitamura A, Iso H, Imano H, Ohira T, Okada T, Sato S, Kiyama M, Tanigawa T, Yamagishi K, Shimamoto T. Carotid intima-media thickness and plaque characteristics as a risk factor for stroke in Japanese elderly men. Stroke 2004;35:2788-94.

22. Underhill HR, Hatsukami TS, Cai J, Yu W, DeMarco JK, Polissar NL, Ota H, Zhao X, Dong L, Oikawa M, Yuan C. A noninvasive imaging approach to assess plaque severity: the carotid atherosclerosis score. AJNR Am J Neuroradiol 2010;31:1068-75.

23. Cai JM, Hatsukami TS, Ferguson MS, Small R, Polissar NL, Yuan C. Classification of human carotid atherosclerotic lesions with in vivo multicontrast magnetic resonance imaging. Circulation 2002;106:1368-73.

24. Saam T, Ferguson MS, Yarnykh VL, Takaya N, Xu D, Polissar NL, Hatsukami TS, Yuan C. Quantitative evaluation of carotid plaque composition by in vivo MRI. Arterioscler Thromb Vasc Biol 2005;25:234-9.

25. Wintermark M, Jawadi SS, Rapp JH, Tihan T, Tong E, Glidden DV, Abedin S, Schaeffer S, Acevedo-Bolton G, Boudignon B, Orwoll B, Pan X, Saloner D. High- resolution CT imaging of carotid artery atherosclerotic plaques. AJNR Am J Neuroradiol 2008;29:875-82.

26. Saba L, Caddeo G, Sanfilippo R, Montisci R, Mallarini G. CT and ultrasound in the study of ulcerated carotid plaque compared with surgical results: potentialities and advantages of multidetector row CT angiography. AJNR Am J Neuroradiol 2007;28:1061-6.

27. Saba L, Sanfilippo R, Sannia S, Anzidei M, Montisci R, Mallarini G, Suri JS. Association between carotid artery plaque volume, composition, and ulceration: a retrospective assessment with MDCT. AJR Am J Roentgenol 2012;199:151-6.

28. Chiu B, Sun J, Zhao X, Wang J, Balu N, Chi J, Xu J, Yuan C, Kerwin WS. Fast plaque burden assessment of the femoral artery using 3D black-blood MRI and automated segmentation. Med Phys 2011;38:5370-84.

29. Zhao H, Wang J, Liu X, Zhao X, Hippe DS, Cao Y, Wan J, Yuan C, Xu J. Assessment of carotid artery atherosclerotic disease by using three-dimensional fast black-blood MR imaging: comparison with DSA. Radiology 2015;274:508-16.

30. Wang J, Yarnykh VL, Yuan C. Enhanced image quality in black-blood MRI using the improved motion-sensitized driven-equilibrium (iMSDE) sequence. J Magn Reson Imaging 2010;31:1256-63.

31. Wang Z, Lu M, Liu W, Zheng T, Li D, Yu W, Fan Z. Assessment of carotid atherosclerotic disease using threedimensional cardiovascular magnetic resonance vessel wall imaging: comparison with digital subtraction angiography. J Cardiovasc Magn Reson 2020;22:18.

32. Wang J, Ferguson MS, Balu N, Yuan C, Hatsukami TS, Börnert P. Improved carotid intraplaque hemorrhage imaging using a slab-selective phase-sensitive inversion-recovery (SPI) sequence. Magn Reson Med 2010;64:1332-40.

33. McNally JS, Kim SE, Mendes J, Hadley JR, Sakata A, De Havenon AH, Treiman GS, Parker DL. Magnetic Resonance Imaging Detection of Intraplaque Hemorrhage. Magn Reson Insights 2017;10:1-8.

34. Bitar R, Moody AR, Leung G, Symons S, Crisp S, Butany J, Rowsell C, Kiss A, Nelson A, Maggisano R. In vivo 3D high-spatial-resolution MR imaging of intraplaque hemorrhage. Radiology 2008;249:259-67.

35. Qiao H, Li D, Cao J, Qi H, Han Y, Han H, Xu H, Wang T, Chen S, Chen H, Wang Y, Zhao X. Quantitative evaluation of carotid atherosclerotic vulnerable plaques using in vivo T1 mapping cardiovascular magnetic resonaonce: validation by histology. J Cardiovasc Magn 
Reson 2020;22:38.

36. Guo M, Cai Y, Yao X, Li Z. Mathematical modeling of atherosclerotic plaque destabilization: Role of neovascularization and intraplaque hemorrhage. J Theor Biol 2018;450:53-65.

37. Willinsky RA, Taylor SM, TerBrugge K, Farb RI, Tomlinson G, Montanera W. Neurologic complications of cerebral angiography: prospective analysis of 2,899 procedures and review of the literature. Radiology 2003;227:522-8.

38. Streifler JY, Eliasziw M, Fox AJ, Benavente OR, Hachinski VC, Ferguson GG, Barnett HJ. Angiographic detection of carotid plaque ulceration. Comparison with surgical observations in a multicenter study. North American Symptomatic Carotid Endarterectomy Trial. Stroke
1994;25:1130-2.

39. D'Onofrio M, Mansueto G, Faccioli N, Guarise A, Tamellini P, Bogina G, Pozzi Mucelli R. Doppler ultrasound and contrast-enhanced magnetic resonance angiography in assessing carotid artery stenosis. Radiol Med 2006;111:93-103.

40. Friedrich JM, Widder B, Schumacher KA, Arlart IP, Hamann H. X-ray diagnosis of intimal ruptures of the internal carotid artery. A pathologico-anatomic controlled study. Rofo 1988;148:111-6.

41. Etesami M, Hoi Y, Steinman DA, Gujar SK, Nidecker AE, Astor BC, Portanova A, Qiao Y, Abdalla WM, Wasserman BA. Comparison of carotid plaque ulcer detection using contrast-enhanced and time-of-flight MRA techniques. AJNR Am J Neuroradiol 2013;34:177-84.
Cite this article as: Cao X, Tang Y, Pan L, Yang J, Wu Y, Geng D, Zhang J. Assessment of carotid atherosclerotic plaque using 3D motion-sensitized driven-equilibrium prepared rapid gradient echo: a comparative study. Quant Imaging Med Surg 2021;11(6):2744-2755. doi: 10.21037/qims-20-869 